\title{
Improvements of stochastic weather data generators for diverse climates
}

\author{
Henry N. Hayhoe* \\ Eastern C ereal and Oilseed Research Centre, Research Branch, Agriculture and Agri-Food C anada, Ottawa, \\ Ontario K1A OC 6, C anada
}

\begin{abstract}
Weather data generators can be used to produce long periods of synthetic weather records from a limited amount of input data. They provide a useful tool for supplementing existing climate data for risk assessment and decision support systems. They also can be adapted for use in climate change studies where only limited data are available on a course spatial grid. A number of studies have demonstrated the failure of data produced with stochastic weather data generators to mimic the statistical properties of observed daily climate data. Differences may be large enough to limit the usefulness of the output data. This study suggests that the lack-of-fit is related to simplifying assumptions incorporated in these models which may be acceptable in some climates but lead to significant discrepancies when applied under very different climate conditions. M ethods are proposed to address these limitations. The challenge related to choosing an appropriate probability distribution for each climate variable is addressed by approximating the probability distribution of the data using an observed frequency distribution. It is becoming increasingly clear that the correlation between climate variables may differ significantly on a seasonal and regional basis. To account for this, the interrelationship between climate parameters is evaluated for each site once every 2 mo. The way the relationship between the wet or dry status of the day and temperature and solar radiation is accounted for is improved in the model. Observed climate data for 3 selected Canadian climatological stations are used to assess the proposed weather data generator. The results indicate an overall improvement in the correspondence between the means, standard deviations, correlations, probability distributions and extreme values of the observed and estimated weather data series when compared with previously reported results. Related parameters such as the length of wet periods, the length of dry periods and the length of frost-free periods are also found to compare favourably with the observed values.
\end{abstract}

KEY WORDS: Markov chain - Stochastic process - Probability distribution · Correlation · Climate change

\section{INTRODUCTION}

With the growing use of computer simulation models in weather sensitive fields such as hydrology and agriculture there is frequently a requirement for weather generators to supplement observed daily climatological data and to provide a way to simulate the long term effects of weather variability. A number of weather data generators have been developed to meet these requirements: WXGEN is the frequently used model for generating daily weather variables that is used in EPIC, the Erosion/Productivity Impact Calculator (Sharpley \& Williams 1990a,b). It is based on the

*E-mail: hayhoeh@em.agr.ca weather data generator developed by Richardson (1981) and Richardson \& Wright (1984). Racsko et al. (1991) developed a weather generator based on the series approach. Semenov \& Porter (1995) have demonstrated the use of this weather generator to forecast crop yields throughout the growing season by combining available observed weather data with generated data for the remainder of the period. More recently Semenov et al. (1998) made a comparison of WGEN described by Richardson \& Wright (1984) and LARS-WG version 2.6 described by Racsko et al. (1991) and Semenov \& Barrow (1997). Greer et al. (1994) used a weather generator with a decision support system to generate scenarios for grain production under both extreme and normal erosion levels on the Canadian 
Prairies. Wilks (1998) has developed a multisite generalization for stochastic generation of daily precipitation.

There is growing interest in the potential usefulness of weather generators in climate change studies. Wilks (1992) proposed linking scenarios from general circulation models (GCM) to local weather characteristics using a weather generator in order to develop weather scenarios. Semenov \& Porter (1995) used a stochastic weather generator to examine the effect of climate variability on estimates of crop yields from the model AFRCWHEAT. They found that changes in climate variability could have more of an effect on yield and yield variability than changes in mean climate. Schubert (1994) and Dubrovsky (1997) have described the use of weather generators to downscale large-scale climate change data produced with GCM to estimate regional climate changes.

A concern with using stochastic generation of weather variables is that they may fail to adequately represent the statistical properties of observed weather series. As pointed out by Semenov et al. (1998), the output of a weather generator should be tested to ensure the quality is acceptable for a given climate and application. WXGEN simulated values have frequently been unrealistic (Hayhoe \& Stewart 1996). Wallis \& Griffiths (1995) found that $30 \mathrm{yr}$ simulated maximum $\left(T_{\mathrm{x}}\right)$ and minimum temperature $\left(T_{n}\right)$, degree-days, monthly and event precipitation $(\mathrm{P})$ were all outside the expected range in 28 to $60 \%$ of the tests. In addition, frost risks were underestimated. Hayhoe (1998b) demonstrated for Canadian conditions that data generated with WXGEN failed to preserve the correlation between weather variables. In a comparison between the weather generators WGEN (Richardson \& Wright 1984) and LARS-WG for sites in Asia, Europe and the USA, Semenov et al. (1998) found that LARS-WG was able to match the observed data much better because it uses semi-empirical distributions which are more flexible than the simple distributions in WGEN. Both generators were subject to errors because they failed to account for differences in the correlation between variables.

The evidence suggests that improved accuracy in weather generators is associated with more accurately representing the daily probability distributions of weather parameters and accounting for the relationship between variables. It is well recognized that correlations between weather variables are significant and vary between locations and seasons (Dubrovsky 1997, Hayhoe 1998a,b). The use of a first-order M arkov chain to model the occurrence of wet and dry days has been identified as a source of error (Schubert 1994, Wallis \& Griffiths 1995, Semenov et al. 1998). Wilks (1999) found that a simple first-order Markov depen- dence was generally appropriate for central and eastern locations but was inadequate for western stations in the US. The general use of simple distributions as probability models for weather variables has been shown to be invalid in many cases (Hayhoe \& Stewart 1996) and a likely source of error (Semenov et al. 1998). The use of a simple normal distribution is inadequate in many cases, particularly for solar radiation (R). The use of semi-empirical distributions as implemented in LARS-WG has been shown to reduce discrepancies between observed and simulated values but they require more parameters. Schubert (1994) has pointed out that one is faced with making a compromise between simplicity in estimating model parameters and adequacy of the model.

The objective of the study is to resolve some of the problems that have been identified in current weather generators and demonstrate that the changes lead to improvements in the generated data for 3 sites across Canada. Starting with the basic modelling approach used in WGEN (Richardson 1981, Semenov et al. 1998), changes are introduced to address current limitations. A first-order Markov chain is replaced with a second-order chain to better represent the probability of wet and dry periods. Simple probability models are replaced with empirical models derived from observed relative frequencies for each location. Changes in correlation between sites and seasons are accounted for (Hayhoe 1998a,b). A series of weather data is generated for each location using the stochastic model and compared with the corresponding observed weather records using graphical and statistical procedures.

\section{METHODS}

Frequently, weather generators include a first-order $M$ arkov chain to generate the occurrence of wet or dry days and a simple probability model, such as a normal distribution, for the magnitude of weather variables (Richardson 1981, Dubrovsky 1997). A dry day is defined here as a day with less than $0.2 \mathrm{~mm}$ of P. A random number generator is used in conjunction with the probability models to generate a random series. When a wet day is generated, the $\mathrm{P}$ amount may be generated according to a skewed normal distribution (Nicks 1974) or a gamma distribution (Schubert 1994, Dubrovsky 1997). Wilks (1999) successfully used a mixed exponential distribution for $P$. The input parameters generally include: monthly relative frequency of wet and dry day sequences, monthly mean, standard deviation and skewness of $P$ and monthly mean and standard deviation of daily $T_{x}, T_{n}$ and $R$.

The weather generator presented by Richardson (1981) uses a continuous multivariate stochastic pro- 
cess to model $T_{x}, T_{n}$ and $R$. The time series of each variable is reduced to a time series of residual elements by subtracting smoothed daily means and dividing by the standard deviations. The means and standard deviations are adjusted for the wet or dry status of the day (Richardson 1981). The residual series is generated with the equation

$$
x_{k, i}(j)=A x_{k, i-1}(j)+B \varepsilon_{k, i}(j)
$$

where $x_{k, i}(j)$ is a $(3 \times 1)$ matrix for Day $i$ of Year $k$ whose elements are the residuals of $T_{x}(j=1), T_{n}(j=2)$ and $R$ $(j=3) ; \varepsilon_{k, i}(j)$ is a $(3 \times 1)$ matrix of independent random components that are normally distributed with a mean of zero and a variance of unity (M atalas 1967); and A and $B$ are $(3 \times 3)$ matrices whose elements are defined such that the new sequences have the desired serial correlation and cross-correlation coefficients. In WXGEN, the version adapted for use in EPIC (Sharpley \& Williams 1990a,b), A and B are not input parameters but are assumed to be fixed. The daily values of the weather variables are derived by multiplying the residuals by the monthly standard deviation and then adding the monthly mean.

In this study the first-order M arkov chain is replaced with a second-order chain. The assumption of a Markov chain of the first-order is that the wet or dry status of the day is dependent only on the state of the previous day, while with a second-order chain it is dependent on the 2 previous days (Hayhoe \& Baier 1974, Wilks 1999). M onthly values for dry and wet day means and standard deviations are input into the simulation. Rather than use the same monthly means and standard deviation for all days in the month as is done in WXGEN (Sharpley \& Williams 1990a,b), a spline interpolation procedure (Press et al. 1992) is used to calculate daily wet and dry day values from the monthly means and standard deviations input into the model. The daily values of the generated weather variables are derived by multiplying the residual series by the corresponding interpolated daily standard deviation and then adding the interpolated daily mean. The interpolated daily wet and dry day values for means and standard deviations derived from observed monthly values are also used to calculate a standardized series from the observed weather data by subtracting the daily mean and dividing by the standard deviation (Richardson 1981). The observed standardized series is used in calculating input parameters used by the weather generator.

Cross and serial correlations for the residual series are calculated on a bimonthly basis (Matalas 1967). Bimonthly is defined here as once in 2 months. The monthly groupings were: January and February, March and April, May and J une, July and August, September and October and finally November and
December. The lag 0 cross-correlation matrix is denoted by $M_{0}$. It consists of elements $\rho_{0}(i, j)$, which are the lag 0 cross-correlation coefficients between variables $\mathrm{i}, \mathrm{j}$. The lag 1 serial correlation matrix is denoted by $M_{1}$. It consists of elements $\rho_{1}(i, j)$, which are lag 1 cross-correlation coefficients between variables $\mathrm{i}, \mathrm{j}$. $T_{x}$ corresponds to an index value of $1, T_{n}$ to a value of 2 and $\mathrm{R}$ to a value of 3 . For example, $\rho_{1}(1,3)$ is the correlation between $T_{x}$ and $R$ lagged by $1 \mathrm{~d}$. The matrix $A$ in Eq. (1) is given by

and

$$
A=M_{1} M_{0}^{-1}
$$

$$
B^{\top}=M_{0}-M_{1} M_{0}^{-1} M_{1}^{\top}
$$

The J acobi transformation of $\mathrm{BB}^{\mathrm{T}}$ is used to solve for $B$ (Press et al. 1992). A matrix $\boldsymbol{v}$, with columns containing the standardized eigenvectors of $\mathrm{BB}^{\top}$, is found such that $\left(\boldsymbol{v}^{\top} B\right)\left(\boldsymbol{v}^{\top} B\right)^{\top}$ is a diagonal matrix in which the the diagonal elements are the eigenvalues of $\mathrm{BB}^{\top}$. Then

$$
B=v\left(v^{\top} B\right)
$$

where $\boldsymbol{v}^{\top} B$ is a diagonal matrix in which the diagonal elements are the square roots of the eigenvalues of $\mathrm{BB}^{\top}$. Automating the calculation of $\mathrm{A}$ and $\mathrm{B}$ is required when bimonthly values are required for a number of locations.

The bimonthly wet and dry day cumulative frequency distributions are calculated from the standardized observed data for $T_{x}, T_{n}$ and $R$. By combining the observations from 2 mo over $30 \mathrm{yr}$, approximately 1800 observations are available to characterize the cumulative frequency distributions. The approach provides an alternative to the requirement of selecting a simple probability model, such as the normal distribution, since it uses the cumulative frequency distribution derived from the observed data. The data are sorted in ascending order. The probability of exceeding any specified level is estimated from the relative frequency that this occurred in the observed data. 101 grid points are used so that the relative frequencies are calculated to 2 decimal places over the full range in probability from 0 to 1 . Cubic spline interpolation is used to derive the continuous cumulative probability distribution for each parameter following algorithms from Press et al. (1992). This procedure was felt to provide an adequate representation of the distribution and meet the requirement to limit the size of the data file. It was found that in some cases it is better to use the logarithm of $\mathrm{P}$ amounts on wet days throughout the generation process and then apply the exponential function to the generated values to obtain $\mathrm{P}$. The distribution of the logarithm of $\mathrm{P}$ on wet days is closer to the normal shape than $\mathrm{P}$ and therefore may facilitate the approximation of the extremes. 
Richardson (1981) notes that the use of Eq. (1) implies that the residuals of $T_{x}, T_{n}$ and $R$ are normally distributed. For locations where this is not a valid assumption, the use of Eq. (1) can lead unfavourable results. For these conditions, a normal score transformation can be used (J ohnson 1987). The normal score transformation converts the observed cumulative probability distribution into a cumulative normal probability distribution for input into Eq. (1). The output from Eq. (1) is then transformed back to the observed cumulative distribution using the inverse of the normal score transformation. The normal score transformation is frequently used in stochastic simulations (Deutsch \& J ournel 1992).

The parameters for the second-order Markov chain are all estimated from relative frequencies of wet and dry day sequences on a monthly basis. Two probabilities are estimated for a first-order chain and 4 for a second-order chain. Random sequences of wet and dry days are generated using a random number generator and assuming the second-order M arkov chain model. Once the wet or dry status of the day is determined, the appropriate cumulative probability distribution is selected and residual elements are generated from it using output from a random number generator. Eq. (1) is used to account for the correlation between variables. The spline interpolated daily wet and dry day values for means and standard deviations are used to produce the actual weather parameters from the generated residual series.

There are a number of physical constraints which must be satisfied by the generated data series. $P$ amounts and $\mathrm{R}$ must be greater than or equal to zero. It is preferable to refine the model to deal with these physical constraints rather than force the values to satisfy the constraints after the data has been generated. At each step in the generating process, the residual series is checked and adjusted as required to maintain the appropriate parameters, such as R, greater than or equal to zero. In the generated data, daily values of $T_{n}$ may happen to be greater than $T_{x}$. This is a contradiction which is usually resolved by adjusting $T_{n}$. For example, in the LAR-WG model this problem is resolved by replacing $T_{n}$ with $T_{x}$ less 0.1 (Semenov et al. 1998). Making adjustments to generated data will change the means, standard deviations and the distribution of the corrected data. One method used to reduce the likelihood of $T_{n}$ being greater than $T_{x}$ is to account for the correlation between $T_{n}$ and $T_{x}$ with Eq. (1). A further refinement is incorporated in the model to avoid changing the value of $T_{n}$. It involves sorting days where $T_{n} \geq\left(T_{x}-5\right)$ and matching higher $T_{n}$ values with the higher $T_{x}$ values.

It can be useful to be able to specify the monthly means and standard deviations of the generated data such that they correspond to observed data or to nearby stations with slightly different monthly means. To do this, the monthly means and standard deviations of daily values for the generated data are calculated and used to evaluate the residual series. A new generated weather series is reproduced using these residual values and specified monthly mean and standard deviation. The procedure is specified by the equation

$$
x_{a}=\left[\left(x_{g}-\bar{x}_{g}\right) / s_{g}+\bar{x}_{a}\right] s_{a}
$$

where $x_{g}$ is the generated value of a daily weather variable such as $T_{x}, T_{n}$ or $R, \bar{x}_{g}$ and $s_{g}$ are the monthly mean and standard deviation of the variable and $x_{a}$ is the adjusted value of a daily weather variable with specified $\bar{x}_{a}$ and $\mathrm{s}_{\mathrm{a}}$. This procedure was used to make minor changes to the generated weather series for $T_{x}$, $\mathrm{T}_{\mathrm{n}}$ and $\mathrm{R}$ so that monthly means and standard deviations corresponded to the observed values. This approach could be used to adjust the means and standard deviations for climate change scenarios.

Three climate stations were selected from different climate zones in Canada: Fredericton $\left(45^{\circ} 55^{\prime} \mathrm{N}\right.$, $\left.66^{\circ} 37^{\prime} \mathrm{W}\right)$, Ottawa $\left(45^{\circ} 23^{\prime} \mathrm{N}, 75^{\circ} 43^{\prime} \mathrm{W}\right)$ and Swift Current $\left(50^{\circ} 16^{\prime} \mathrm{N}, 107^{\circ} 44^{\prime} \mathrm{W}\right)$. Thirty years (1960 to 1989 ) of observed daily data for each station are input and used in a computer program to produce all the required inputs for the weather data generator including transition probabilities for the second-order $M$ arkov chain for wet and dry days, daily means, daily standard deviations, residual values, correlations, A and B matrices for Eq. (1), and cumulative probability distributions of observed daily $T_{x}, T_{n}, P$ and $R$. Both wet and dry day means and standard deviations were calculated on a monthly basis and interpolated using splines to generate daily values. The residual series generated with these daily values was used to estimate monthly lag 0 and lag 1 cross-correlation coefficients and to calculate bimonthly values of the $A$ and $B$ matrices in Eq. (1). Bimonthly cumulative frequency distributions were calculated for wet day $\mathrm{P}$ and for wet and dry day values of $T_{x}, T_{n}$ and $R$, using the residual series. The cumulative frequency distributions provided the probability models in this computer implementation. The inputs were used by the weather data generator to produce $30 \mathrm{yr}$ of data for each station.

Throughout the analysis the t-test was used to test the significance $(p \leq 0.05)$ of differences in means; the $F$-test was used to test the significance $(p \leq 0.05)$ of differences in standard deviations; and the KolmogorovSmirnov test was used to test the significance $(p \leq 0.05)$ of differences in the distribution. The unequal variance t-test was used with variances estimated from the data. The fact that some of the variables might not al ways be normally distributed was overlooked for $T_{x}, T_{n}$ and $R$ and for frost-free periods, since it has been found that 
in many cases one can still use the normal distributionbased tests if the size of the sample is large enough. Based on the central limit theorem, the shape of the distribution of a statistic, such as the mean derived from a sample, approaches the normal shape even if the distribution of the variable in question is not normal. Values of $P$ for wet days were transformed using the logarithm function before applying the t-test and F-test. The observed and generated weather data series were assumed to be independent because of the random nature of the stochastic generation process. This was confirmed by checking the correlation between variables in the generated and observed series. The Kolmogorov-Smirnov test is a nonparametric test. The 2-distribution form of the test was used to test the null hypothesis that the observed and generated series were drawn from the same distribution. The 1-distribution form of the test was used to test the null hypothesis that the observed or generated series was drawn from the normal distribution. Estimation of the parameters for the normal distribution is not accounted for with the 1-distribution Kolmogorov-Smirnov test, but this is not considered to be a problem because of the large number of data points in the series (approximately 900). STATISTICA (StatSoft, Inc. 1997) was used to provide statistical analyses of results.

\section{RESULTS}

The weather data generated in this study have statistical properties which are generally not significantly different from the observed weather data. Table 1 pro-

Table 1. Results of statistical tests showing the number of months with significant differences $(p \leq 0.05)$ between means, standard deviations and probability distributions for observed and generated weather data series

\begin{tabular}{|lccc|}
\hline Variable & Fredericton & Ottawa & Swift Current \\
\hline Precipitation & & & \\
Mean & 0 & 0 & 0 \\
Variance & 0 & 0 & 3 \\
Distribution & 0 & 0 & 0 \\
Maximum temperature & 0 & 0 & 0 \\
Mean & 0 & 0 & 0 \\
Variance & 0 & 0 & 0 \\
Distribution & 1 & & \\
Minimum temperature & 0 & 0 & 0 \\
Mean & 0 & 0 & 0 \\
Variance & 0 & 0 & 2 \\
Distribution & 2 & & \\
Solar radiation & & 0 & 0 \\
Mean & 0 & 0 & 0 \\
Variance & 0 & 0 & 1 \\
Distribution & 0 & & \\
\hline
\end{tabular}

vides a summary of how well the generated and observed data correspond. These results give fewer significant differences than in previous published results using WXGEN (Hayhoe \& Stewart 1996).

The stochastic model generated values for $P$ and $R$ which consistently satisfied physical constraints. Generated values of $P$ and $R$ were greater than or equal to zero. The value of $R$ remained below the maximum possible clear day value. The number of times $T_{n}$ was greater than $T_{x}$ varied generally between 50 and 100 over the $30 \mathrm{yr}$ simulation period. As indicated, rather than arbitrarily forcing $T_{n}$ to be less than $T_{x}$, days where $T_{n} \geq\left(T_{x}-5\right)$ were sorted and then higher $T_{n}$ values were matched with the higher $T_{x}$ values. This was done on a monthly basis and on average it involve about $8 \%$ of the generated temperatures. Using this approach generally eliminated the cases where $T_{n}$ was greater than $T_{x}$ and when it did not there were at most a few cases where the values of $T_{n}$ and $T_{x}$ were close. These few remaining cases were eliminated by replacing $T_{n}$ with $T_{x}$ less 0.1 (Semenov et al. 1998).

There were few significant differences in the monthly standard deviation of daily values of $P$, with a total of 3 occurring at Swift Current. It is interesting to note that there were no corresponding significant differences in the distribution of P. At Ottawa, none of the generated series for $T_{x}, T_{n}$ or $R$ had significantly different distributions from the observed, but at Fredericton there was 1 month (November) where the distribution of $T_{x}$ was significantly different and 2 months ( $M$ arch and October) where the distribution of the generated series for $T_{n}$ had significant differences. At Swift Current there were no months where the distribution of $T_{x}$ was significantly different, 2 months ( $M$ arch and A pril) where the distribution of the generated series for $T_{n}$ had significant differences and 1 month (October) where the distribution of the generated series for $\mathrm{R}$ had significant differences. Significant differences most frequently occurred during periods of seasonal change.

Fig. 1 provides a graphical comparison of selected frequency distributions of generated and observed series. These results are typical of what was found. Fig. 1a,b show the correspondence for the frequency distribution for $\mathrm{P}$ during the month of May at Ottawa and Swift Current. Differences were occasionally as large as $12 \mathrm{~mm}$ but it was felt that they could be attributed to natural variability. The differences in the distributions were not found to be statistically significant. Fig. 1c,d illustrate the frequency distribution of $T_{n}$ in March at Fredericton and of $T_{x}$ in April at Swift Current respectively. The observed frequency distribution in Fig. 1c was found to be significantly different from the normal distribution and from the distribution of the generated series. In contrast, the distribution of the generated series was not significantly different from 


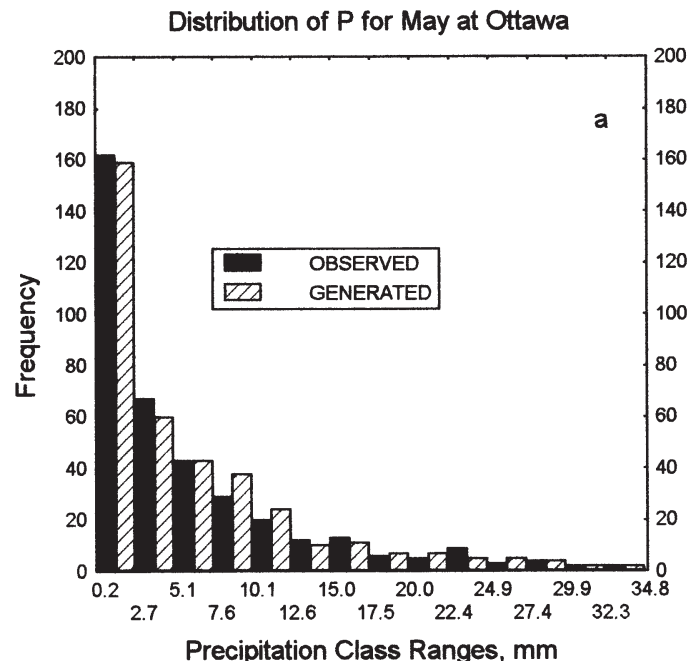

Precipitation Class Ranges, $\mathrm{mm}$

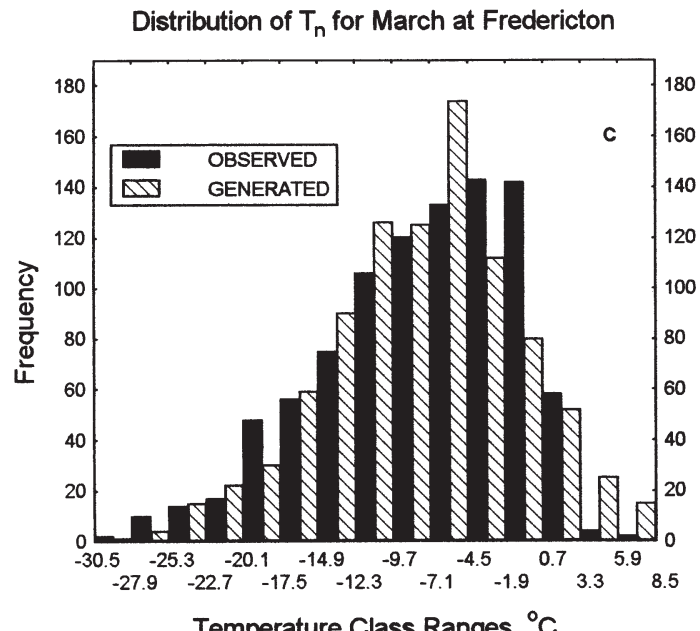

Temperature Class Ranges, ${ }^{\circ} \mathrm{C}$

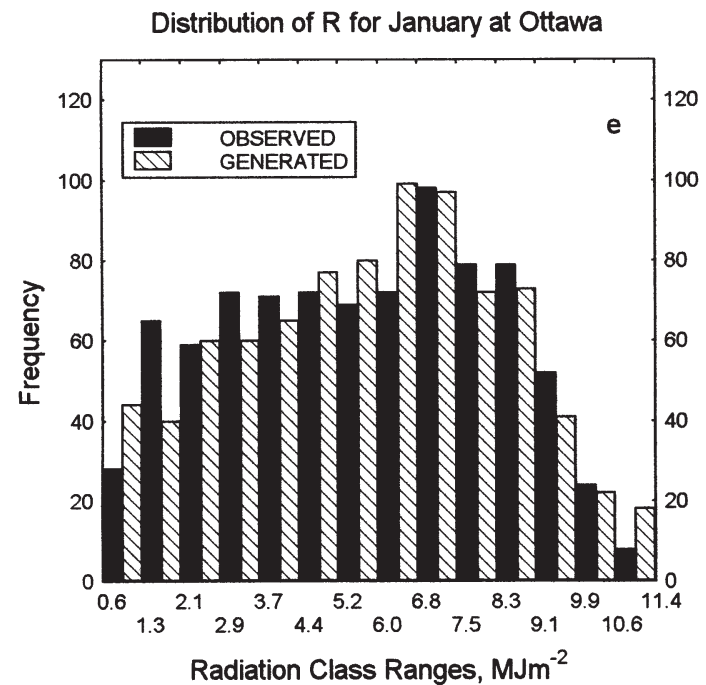

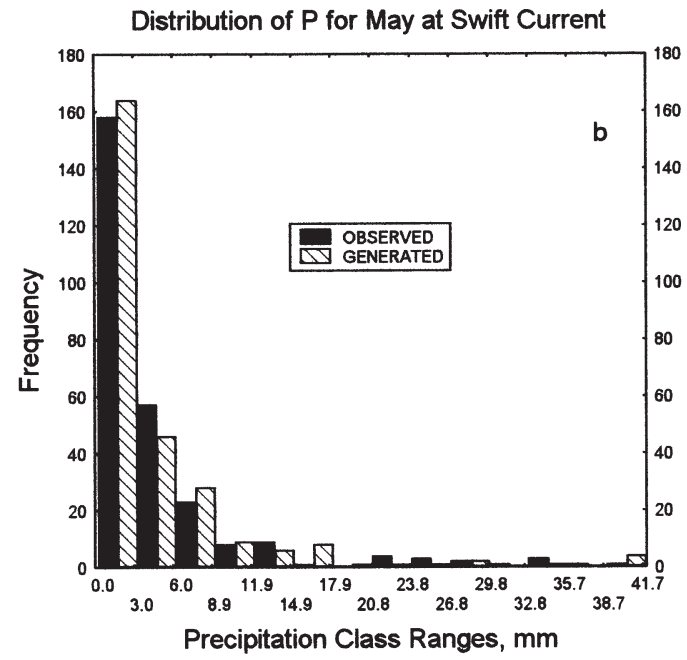

Distribution of $T_{x}$ for April at Swift Current

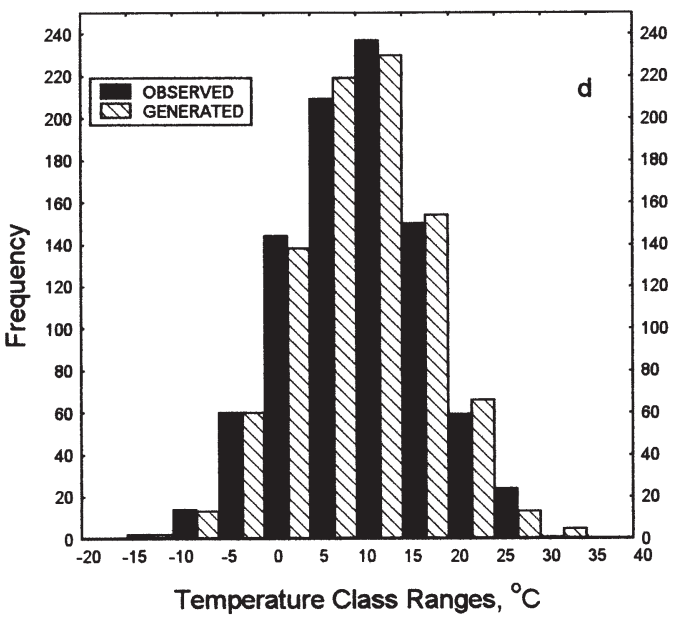

Distribution of $\mathrm{R}$ for August at Ottawa

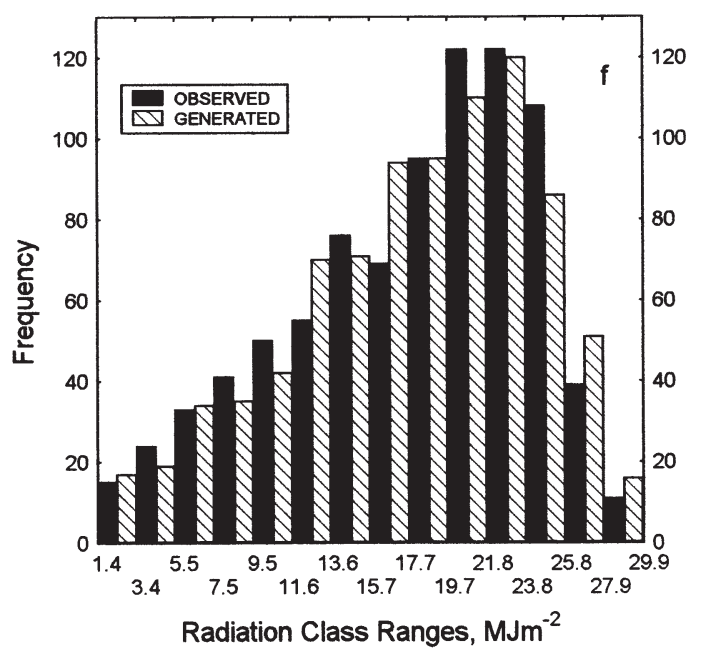

Fig. 1. Frequency distribution derived from observed and generated data for: (a) P for M ay at Ottawa, (b) P for M ay at Swift Current, (c) $T_{n}$ for M arch at Fredericton, (d) $T_{x}$ for A pril at Swift Current, (e) R for J anuary at Ottawa and (f) R for August at Ottawa 
the normal distribution. These differences could result from using distributions calculated for 2 months (March and April) in a period of seasonal change. In contrast, for $T_{x}$ in A pril at Swift Current, the observed frequency distribution in Fig. 1d was not found to be significantly different from the normal distribution or from the distribution of the generated series. Fig. le,f show the distribution of R at Ottawa for J anuary and August. The observed frequency distribution of $R$ in $J$ anuary in Fig. le was not found to be significantly different from the distribution of the generated series. In August (Fig. If) the observed distribution appeared skewed but not significantly different from the distribution of the generated distribution. It was found to be significantly different from the normal distribution.

It has been suggested that extremes and the tails of distributions may have more impact on the use of the generated data than statistical parameters such as means and standard deviations. Wilks (1999) used graphical presentations to illustrate the extreme-value characteristics of a number of probability models. For example, 1-to-1 graphs of largest observed daily precipitation were plotted against the largest modelled daily precipitation. A similar approach is used in Fig. 2 to examine the extreme-value characteristics of observed and generated series for monthly values $\mathrm{P}$, $T_{x}, T_{n}$ and R. Fig. 2a is a plot of the largest observed against the largest generated $P$ for each month for all 3 locations combined. There is considerable scatter in the data but it fits the 1-to- 1 line well. The means of the observed and generated monthly extreme values of $P$ were 48.7 and $45.9 \mathrm{~mm}$ respectively and the corresponding standard deviation of the difference between the observed and generated was $10.9 \mathrm{~mm}$. Fig. 2b illustrates the same results for $\mathrm{R}$. There is an excellent correspondence between the extremes in the generated and observed series. The means of the observed and generated monthly extreme values of $\mathrm{R}$ were both

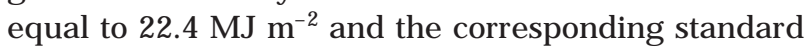
deviation of the differences was $0.7 \mathrm{MJ} \mathrm{m}^{-2}$. Fig. $2 \mathrm{c}, \mathrm{d}$ illustrate the strong 1-to-1 correspondence between lowest and largest extreme values for observed and generated $T_{x}$ respectively. The means of the observed and generated monthly extreme values of $\mathrm{T}_{\mathrm{x}}$ were 26.4 and $26.5^{\circ} \mathrm{C}$ for the largest and -7.2 and $-7.4^{\circ} \mathrm{C}$ for the lowest respectively. The corresponding standard deviations of the differences between the observed and generated monthly extreme values of $\mathrm{T}_{\mathrm{x}}$ were $2.3^{\circ} \mathrm{C}$ for the lowest and $2.2^{\circ} \mathrm{C}$ for the largest. Fig. 2e, f illustrate the 1-to- 1 correspondence between lowest and largest extreme values for observed and generated $T_{n}$ respectively. The means of the observed and generated monthly extreme of $T_{n}$ were 13.6 and $13.9^{\circ} \mathrm{C}$ respectively for the largest and $-17.3^{\circ} \mathrm{C}$ for both the lowest observed and generated extremes. The corresponding standard deviations of the differences between the observed and generated monthly extremes of $T_{n}$ were $2.4^{\circ} \mathrm{C}$ for the lowest and $2.8^{\circ} \mathrm{C}$ for the largest.

It is important to look at how well some derived climate indices are reproduced in the generated data. Fig. 3 shows results for frost-free period. There is more random variability in the observed distribution because it is based on 30 yearly values rather than the approximately 900 values used to characterize the monthly distribution of weather variables such as $T_{x}$. The observed frequency distribution of the frost-free period was found to be significantly different at Fredericton but not significantly different at Ottawa and Swift Current from the generated distribution (Fig. 3). The lack-of-fit at Fredericton can be associated with the fact that the mean frost-free period for the generated series was found to be significantly different from the observed. At Ottawa and Swift Current, no significant differences were found between observed and generated frost-free period means or standard deviations.

Another concern is how well the frequency and length of wet or dry periods correspond to observed frequencies. Semenov et al. (1998) have opted to use observed wet and dry day distributions in LARS-WG because of concern over the lack-of-fit of the first-order Markov chain. Wilks (1999) discussed the use of higher-order and hybrid-order M arkov chains as alternatives to the first-order chains. The frequencies presented here are derived from the M arkov chain model, which in this case is assumed to be second-order. The distribution of the length of wet periods is illustrated in Fig. 4 for all 3 sites. The frequency of wet periods dropped quickly as the number of days in the period increases. These figures show the excellent correspondence between observed and generated series which resulted from using the second-order Markov chain model. There were no significant differences between the distributions at either Fredericton, Ottawa or Swift Current. Table 2 provides an indication of the close agreement between the observed and generated means and standard deviations. The mean length in days of wet periods did not vary much between locations, with a high of $2.11 \mathrm{~d}$ at Ottawa and a low of $1.90 \mathrm{~d}$ at Swift Current.

Table 2 also contains results for dry periods. The mean length of dry day periods varied more between locations, with a low of $2.77 \mathrm{~d}$ at Fredericton and a high of $4.31 \mathrm{~d}$ at Swift Current. There was also greater variation in the standard deviation of dry periods with a low of 2.38 at Ottawa and a high of 4.51 at Swift Current. The graphs of the distributions are not presented, but the Kolmogorov-Smirnov test did not indicate significant differences $(p \leq 0.05)$ between observed and generated distributions at either Fredericton, Ottawa or Swift Current. 

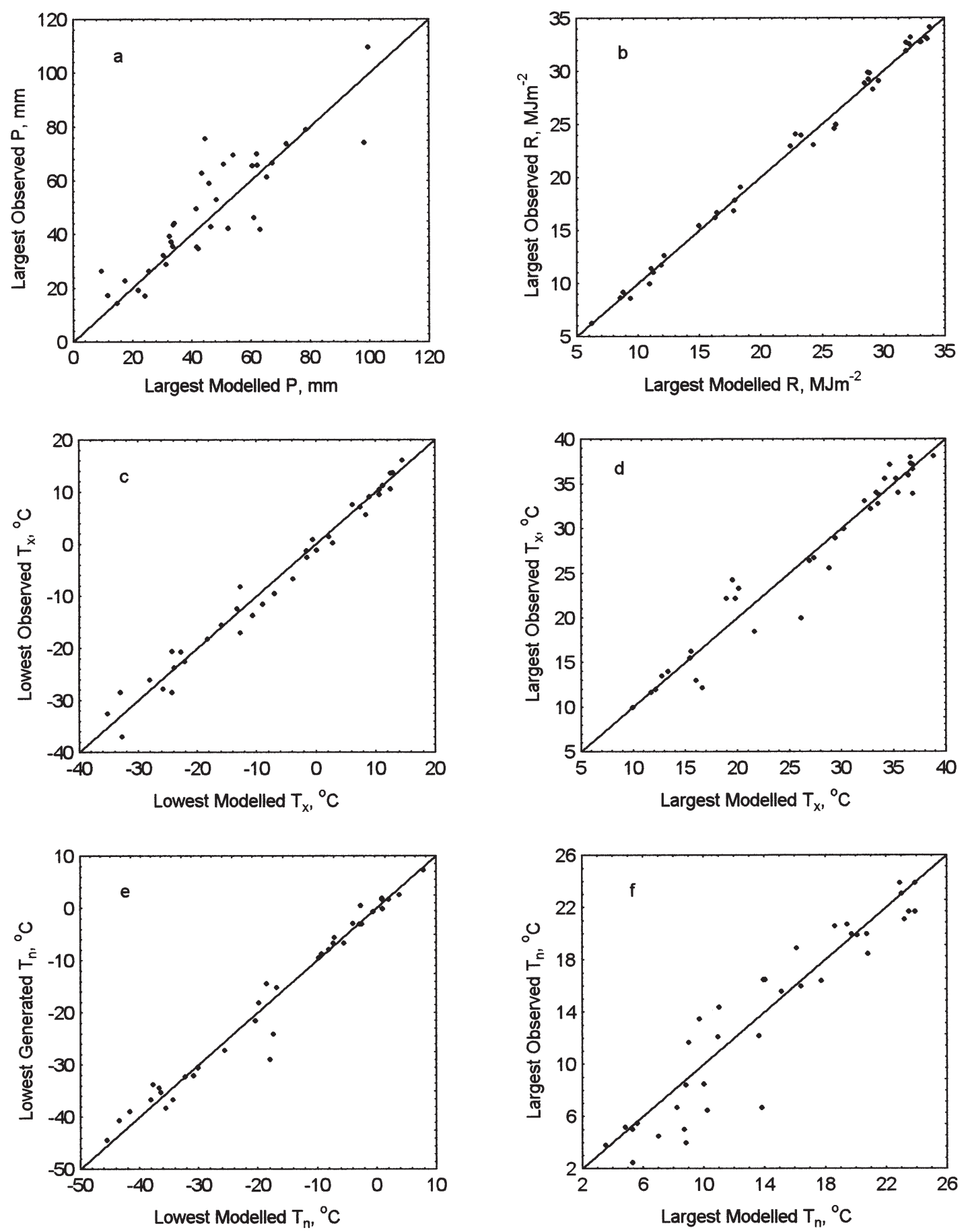

Fig. 2. One-to-one relationship between the monthly extreme-values of observed and generated weather variables at Fredericton, Ottawa and Swift Current combined for: (a) largest daily $P$, (b) largest daily $R$, (c) lowest daily $T_{x}$, (d) largest daily $T_{x}$, (e) lowest daily $T_{n}$ and (f) largest daily $T_{n}$

The relationship between $T_{x}, T_{n}$ and $R$ for the generated data is controlled by the values in the $A$ and $B$ matrices in Eq. (1). Hayhoe (1998a) showed that monthly values of $A$ and $B$ estimated using the observed weather data vary regionally and seasonally. Elements in these matrices have been shown to vary substantially both in magnitude and sign depending on the location and month. Figs. $5 \& 6$ illustrate the correspondence of the correlation between weather variables in the observed and stochastically generated data series at 

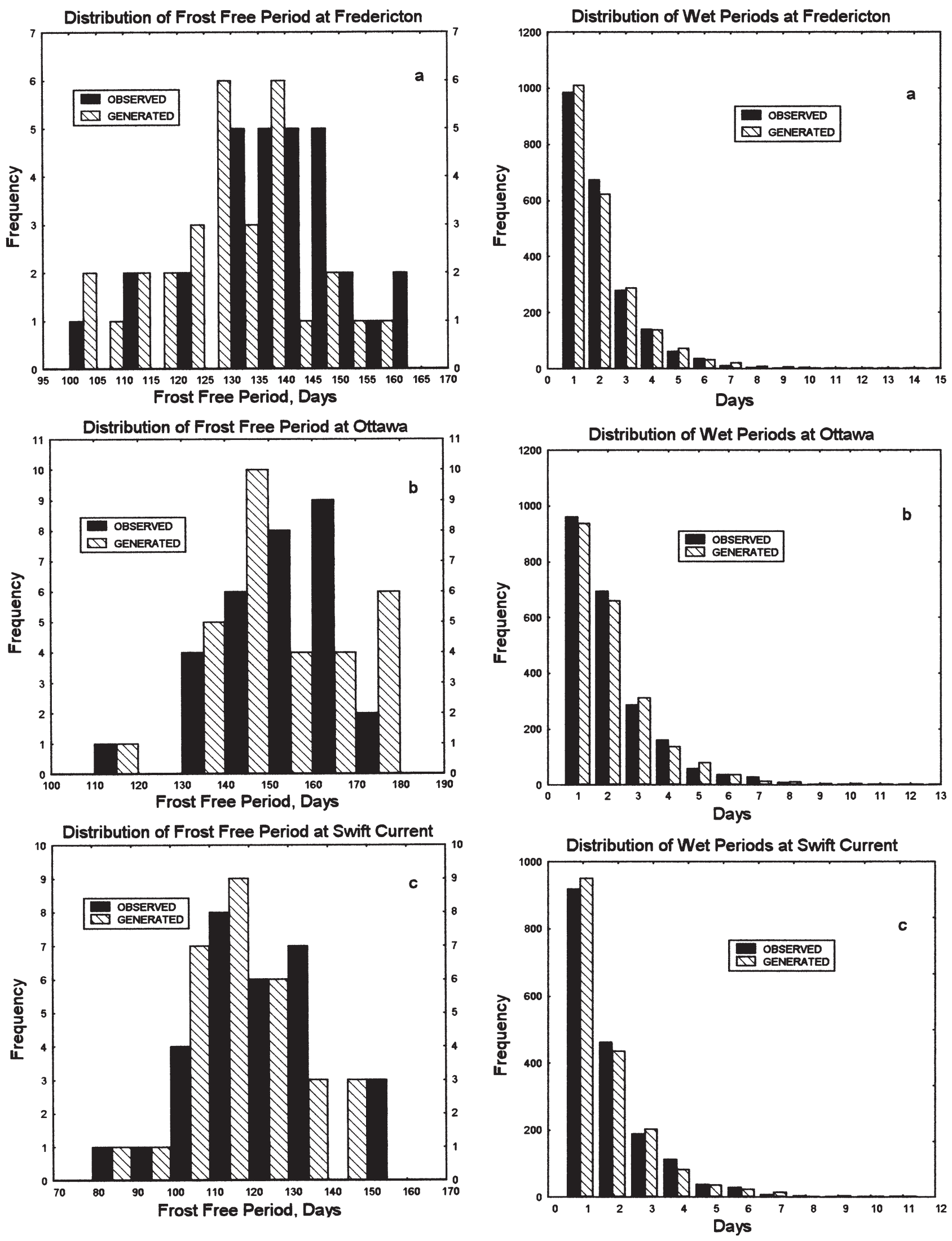

Fig. 3. Frequency distribution derived from observed and generated data for frost-free period: (a) at Fredericton, (b) at Ottawa and (c) at Swift Current

Fig. 4. Frequency distribution derived from observed and generated data for the length of wet periods in days: (a) at Fredericton, (b) at Ottawa and (c) at Swift Current 
Ottawa and Swift Current respectively. Every correlation in Figs. $5 \& 6$ with magnitude greater than 0.07 was found to be significant $(p \leq 0.05)$. The generated data were derived using bimonthly values of $\mathrm{A}$ and $\mathrm{B}$. The observed weather showed seasonal and regional variations in the correlation between all parameters. The generated data gave correlations that correspond well with the value from the observed data (Figs. $5 \& 6$ ). It

Table 2. M eans and standard deviations for wet and dry periods

\begin{tabular}{|c|c|c|c|c|c|c|c|c|}
\hline \multirow[t]{3}{*}{ Site } & \multicolumn{4}{|c|}{ Wet period $(\mathrm{d})$} & \multicolumn{4}{|c|}{ Dry periods $(\mathrm{d})$} \\
\hline & \multicolumn{2}{|c|}{ Mean } & \multicolumn{2}{|c|}{ Standard deviation } & \multicolumn{2}{|c|}{ Mean } & \multicolumn{2}{|c|}{ Standard deviation } \\
\hline & Obs & Gen & Obs & Gen & Obs & Gen & Obs & Gen \\
\hline Fredericton & 2.05 & 2.05 & 1.42 & 1.4 & 2.92 & 2.92 & 2.43 & 2.36 \\
\hline Ottawa & 2.11 & 2.12 & 1.46 & 1.45 & 2.77 & 2.85 & 2.38 & 2.43 \\
\hline Swift Current & 1.9 & 1.84 & 1.3 & 1.25 & 4.31 & 4.42 & 4.51 & 4.32 \\
\hline
\end{tabular}
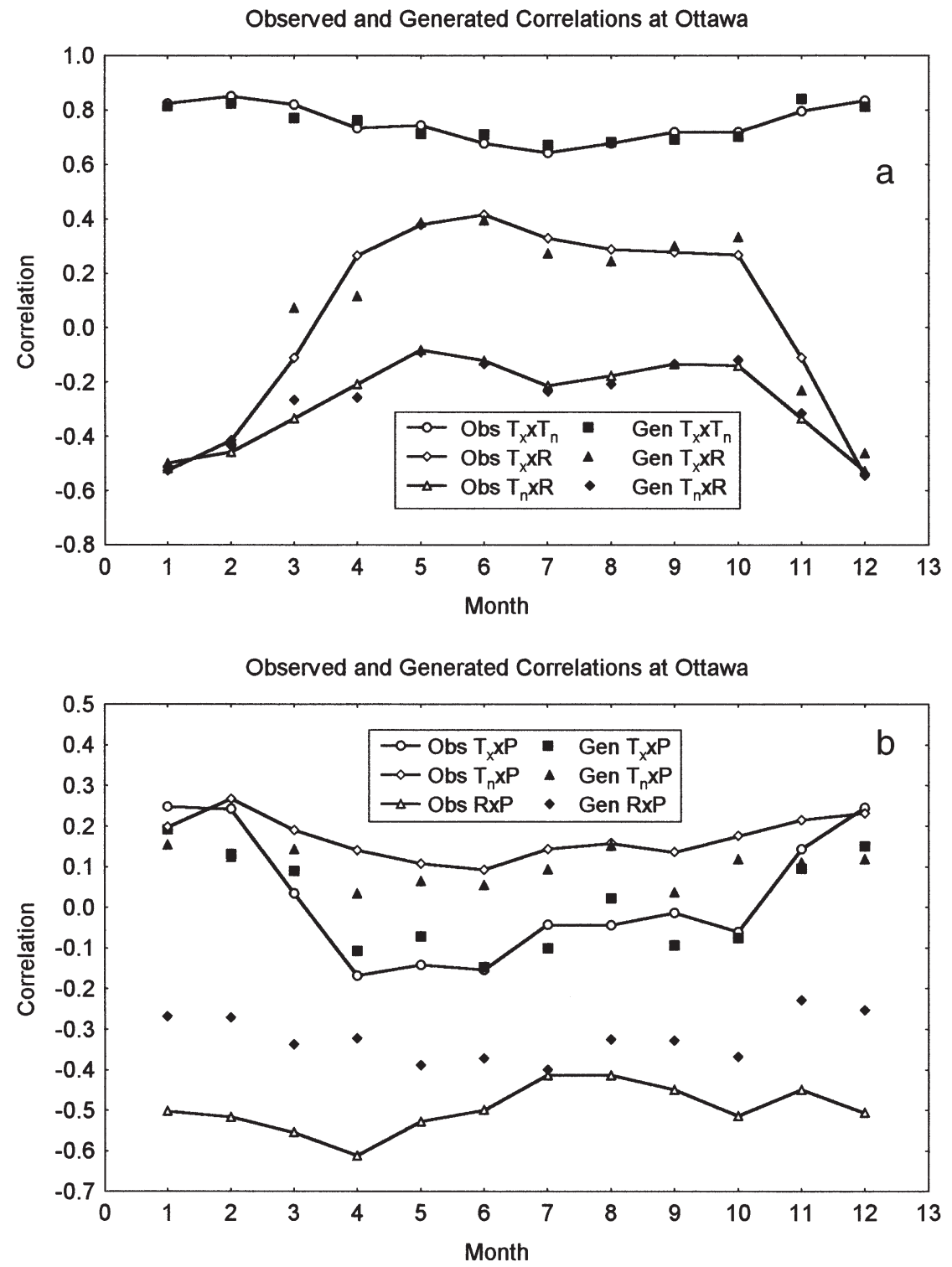

Fig. 5. Correlations derived on a monthly basis from observed and generated data at Ottawa for: (a) $T_{x}$ with $T_{n}, T_{x}$ with $R$, and $T_{n}$ with $R$, and (b) $T_{x}$ with $P, T_{n}$ with $P$ and $R$ with $P$ 
has been shown (Hayhoe 1998b) that models, such as WXGEN, which do not account for seasonal or regional variation in $\mathrm{A}$ and $\mathrm{B}$ produce generated data with relatively constant correlation between variables for all the stations and seasons. This is to be expected since the values in the matrices $A$ and $B$ are held constant.

The highest observed correlation of 0.88 occurred at Swift Current in February (Fig. 6a). Figs. $5 a \& 6$ a show that the generated data gave correlations between $T_{x}$ and $T_{n}$ throughout the year that corresponded well with the value from the observed data. Correlations between $T_{x}$ and $T_{n}$ were always significant $(p \leq 0.05)$ both in the observed and generated data for Ottawa and Swift Current. The seasonal variation in the correlation was greater at Swift Current than at Ottawa. Using bimonthly values of $A$ and $B$ produced generated data which accounted for the seasonal variation in the observed relationship between $T_{x}$ and $\mathrm{R}$. For example, at Ottawa in J anuary the observed correlation was -0.53 and the corresponding value for generated data was -0.52 (Fig. 5a). The corresponding values for Ottawa in J une were 0.42 and 0.40 (Fig. 5a). The observed correlation between $T_{x}$ and $R$ was always negative from December through February, suggesting higher radiation on cooler days, while it was always positive from April through October, suggesting
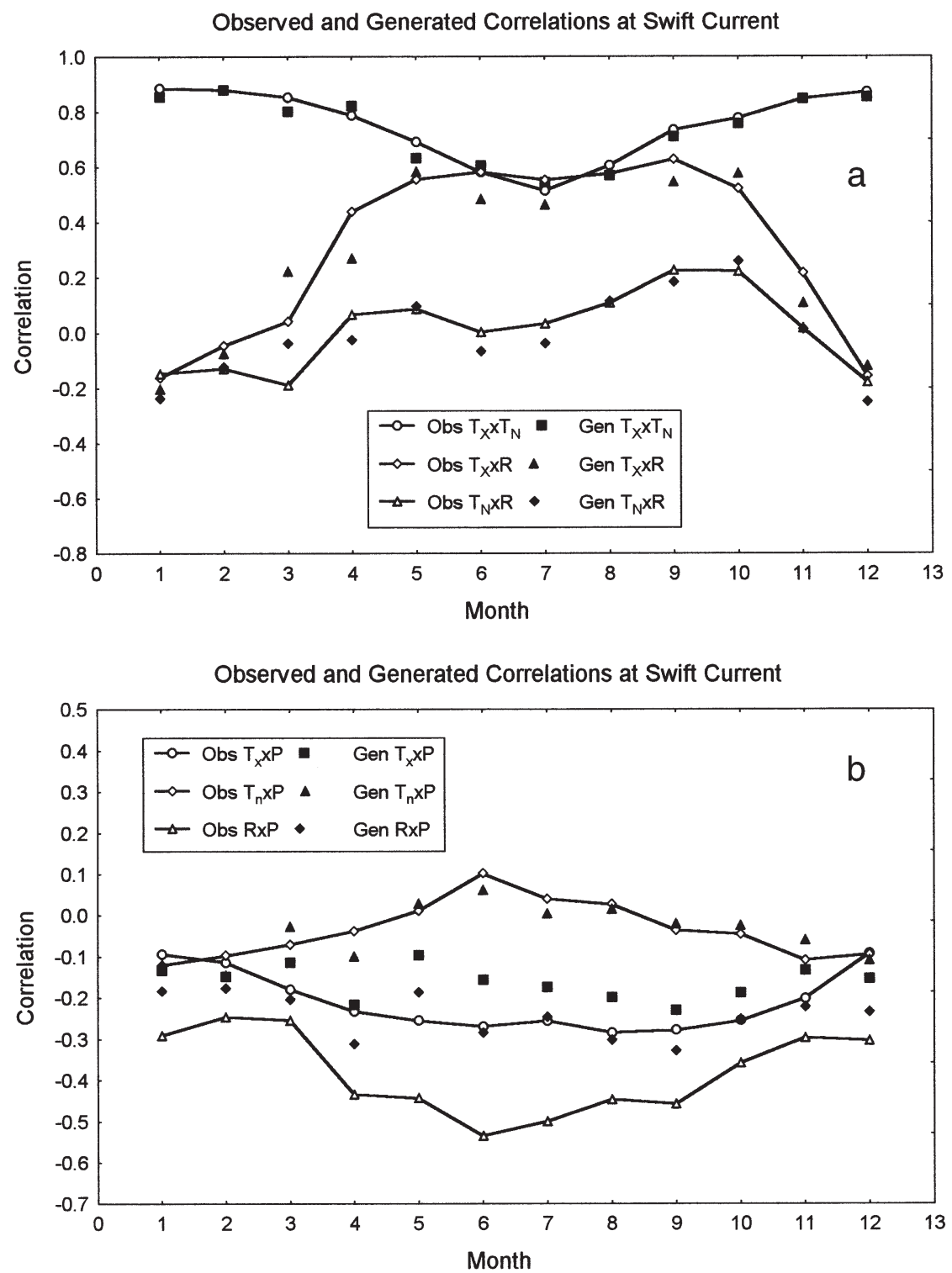

Fig. 6. Correlations derived on a monthly basis from observed and generated data at Swift Current for: (a) $T_{x}$ with $T_{n}, T_{x}$ with $R$, and $T_{n}$ with $R$, and (b) $T_{x}$ with $P, T_{n}$ with $P$ and $R$ with $P$ 
higher radiation on warmer days. The poorer correspondence between correlations for $T_{x}$ and $R$ in the observed and generated data during $M$ arch and April (Figs. 5a \& 6a) may result from using bimonthly rather than monthly values for $A$ and $B$ during seasonal change (Hayhoe 1998a,b). There were also regional differences, as illustrated by the higher correlations at Swift Current in J uly and A ugust than at Ottawa (Figs. $5 a \& 6 a)$. There was less seasonal variation in the correlation between $T_{n}$ and $R$ than with $T_{x}$. The correlations remained negative throughout the year at Ottawa (Fig. 5a). The magnitude of the correlation was generally lower at Swift Current and remained positive from A pril through November (Fig. 6a).

In the observed data, the correlation between $T_{x}$ and $P$ was positive from November through February and negative from April through October at Ottawa (Fig. 5b). The corresponding correlation was always negative at Swift Current (Fig. 6b). The weather data generated using observed wet and dry day parameters (Richardson 1981) followed the same seasonal pattern as the observed (Figs. $5 b \& 6 b$ ). The observed data showed that the magnitude of the correlation was frequently higher between $P$ and $T_{n}$ than between $P$ and $T_{x}$. At Ottawa, the correlation between $P$ and $T_{n}$ was consistently positive throughout the year. The relationship between $P$ and $T_{n}$ is frequently ignored in weather generators (Hayhoe \& Stewart 1996).

The correlation between $\mathrm{R}$ and $\mathrm{P}$ was always negative for the observed data. Correlations between $\mathrm{P}$ and $R$ were always significant $(p \leq 0.05)$ both in the observed and generated data for Ottawa and Swift Current. The weather data generated here used observed wet and dry day means and standard deviations. The resulting correlations tended to follow the seasonal and regional pattern of the observed data but generally underestimated the magnitude of the correIation (Figs. $5 b \& 6 b$ ). This may be related to the fact that the model does not account for the possible effect of the amount of precipitation on solar radiation. It only considers whether or not a given day is classified as wet or dry.

The generated data also maintains the lag 1 serial correlations, including the seasonal variation. Lag 1 correlations are contained in the matrix $\mathrm{M}_{1}$, whose elements are $\rho_{1}(i, j)$, the lag 1 cross-correlation coefficients between variables $i, j$ where $T_{x}$ corresponds to an index value of $1, T_{n}$ to a value of 2 and $R$ to a value of 3. Eq. (6) indicates the correlations for Ottawa in J anuary and February and Eq. (7) gives the corresponding values for J uly and August for both the observed series and the generated series. The matrices confirm the excellent agreement between the observed and generated series as well as showing the substantial seasonal change.

$$
M_{1}^{\text {obs }}=\left[\begin{array}{rrr}
0.62 & 0.61 & -0.37 \\
0.64 & 0.68 & -0.42 \\
-0.22 & -0.26 & 0.15
\end{array}\right] \quad M_{1}^{\text {gen }}=\left[\begin{array}{rrr}
0.60 & 0.58 & -0.33 \\
0.63 & 0.65 & -0.39 \\
-0.20 & -0.22 & 0.15
\end{array}\right]
$$

$$
M_{1}^{\text {obs }}=\left[\begin{array}{rrr}
0.55 & 0.47 & 0.09 \\
0.67 & 0.65 & -0.09 \\
0.02 & 0.01 & 0.12
\end{array}\right] \quad M_{1}^{\text {gen }}=\left[\begin{array}{rrr}
0.58 & 0.52 & 0.05 \\
0.69 & 0.68 & -0.10 \\
0.01 & 0.02 & 0.09
\end{array}\right]
$$

\section{DISCUSSION AND CONCLUSION}

The results indicate an overall improvement in the correspondence between observed and estimated weather data series when compared with previously reported results (Hayhoe \& Stewart 1996, Hayhoe 1998a,b, Semenov et al. 1998). For example, at Ottawa the number of months with significant differences between observed and WXGEN generated distributions was 3 for $T_{x}, 6$ for $T_{n}, 9$ for $P$ and 12 for $\mathrm{R}$ (Hayhoe $\&$ Stewart 1996) compared with no significant differences for Ottawa indicated in Table 1. A further test of the model is the correspondence between observed and generated extreme values. The results indicate that there was in general a good 1-to-1 relationship between extremes for $P, T_{n}, T_{x}$ and $R$. The distributions derived from generated data for frost-free periods at Ottawa and Swift Current and for wet or dry periods at all locations were not found to differ significantly from the observed. This is attributable to a number of improvements in the model. These include using observed monthly means and standard deviations of wet and dry day $T_{x}, T_{n}$ and $R$ and generating daily values from the monthly values using spline interpolation. Another improvement was the use of bimonthly observed distributions for $\mathrm{P}$ and separate observed distributions for $T_{x}, T_{n}$ and $R$ for wet and dry days. Further, replacing the conventional first-order Markov chain with a second-order chain provided for a more reliable estimate of wet and dry day sequences. Correlation between variables was accounted for using local values for $A$ and $B$ calculated on a bimonthly basis.

It is difficult to assess the importance of some of the differences between observed and generated weather data. Since the weather data generator is a stochastic model, some of the differences can be attributed to natural variability of weather that would occur even if the model were a perfect representation of the real climate. Other differences may result from limitations and simplifying assumptions in the model. Statistical significance tests provide some indication of whether 
or not differences such as those illustrated in the graphs of frequency distributions (Figs. 2, 3\& 4) are the result of natural variability or limitations of the model. The indication is that in many cases one is justified in concluding that differences between observed and generated data could result from the inherent variability of weather. Differences which result from limitations in the model are a concern and should be carefully evaluated for a given climate to ensure that the generated data are acceptable for the intended application (Semenov et al. 1998).

Lack-of-fit of the statistical properties of the generated with the observed series can be attributed to a number of factors. It is felt that the model as presented here addresses many, but not all the potential sources of error. It is very difficult to account for the daily change in the statistical properties of the weather data particularly during times of seasonal change. It becomes a trade off between having enough observed data to properly characterize means, standard deviation, distributions and correlations and not masking the changes in these values over time. A longer period of record could improve the characterization of the climate. For example, bimonthly rather than monthly values were used for correlations, for $A$ and $B$ and for probability distributions. Although the use of relative frequencies and spline interpolation for the observed distributions could be a source of errors, it may be preferable in situations where there is not an appropriate simple distribution. Using observed data, to generate a probability distribution could be a problem if there were significant errors in the observed data as was suggested by Semenov et al. (1998). The data used here are of the highest quality, with very few missing observations. They were empirically checked for obvious outliers. Using A and B matrices to account for the correlation between residual series with different distributions which may be significantly different from the normal distribution may be a source of errors (Matalas 1967), although the approach seems fairly robust. For Swift Current, where some distributions were significantly skewed, the results were improved by using the normal score transformation. A further source of error could be the requirement to force the generated data to satisfy the physical constraints of the system. Refining the model to deal with these physical constraints rather than forcing the values to satisfy the constraints can improve the generated data.

Acknowledgements. This is ECORC Contribution No. 991431.

\section{LITERATURE CITED}

Deutsch VD, J ournel AG (1992) GSLIB geostatistical software library and user's guide. Oxford University Press, Oxford

Editorial responsibility: Laurence Kalkstein, Newark, Delaware, USA
Dubrovsky M (1997) Creating daily weather series with use of the weather generator. Environmetrics 8:409-424

Greer J E, Falk S, Greer KJ , Bentham MJ (1994) Explaining and justifying recommendations in an agriculture decision support system. Comp Elect Agric 11:195-214

Hayhoe HN (1998a) Statistical relationship between weather variables in observed and stochastically generated data series. In: Preprints for the 14th Conference on Probability and Statistics in the Atmospheric Sciences, 78th AMS Annual Meeting, 11-16 January 1998, Phoenix, AZ. A merican Meteorological Society, Boston, MA, p 60-65

Hayhoe HN (1998b) Relationship between weather variables in observed and WXGEN generated data series. A gric For Meteorol 90:203-214

Hayhoe HN, Baier W (1974) Markov chain model for sequences of field workdays. Can J Soil Sci 54:I37-148

Hayhoe HN, Stewart DW (1996) Evaluation of CLIGEN and WXGEN weather data generators under Canadian conditions. Can Water Resour J 21(1):53-67

J ohnson M (1987) Multivariate statistical simulation. John Wiley \& Sons, New York

Matalas NC (1967) Mathematical assessment of synthetic hydrology. Water Resour Res 3:937-945

Nicks AD (1974) Stochastic generation of the occurrence, pattern, and location of maximum amount of daily rainfall. In: Proceedings of the Symposium on Statistical Hydrology, Tucson, AZ. US Department of Agriculture Misc Publ 1275, p 154-171

Press WH, Teukolsky SA, Vetterling WT, Flannery BP (1992) Numerical recipes in FORTRAN, the art of scientific computing, 2nd edn. Cambridge University Press, Cambridge

Racsko P, Szeidl L, Semenov M (1991) A serial approach to local stochastic weather models. Ecol Model 57:27-41

Richardson CW (1981) Stochastic simulation of daily precipitation, temperature, and solar radiation. Water Resour Res 17(1):182-190

Richardson CW, Wright DA (1984) WGEN : a model for generating daily weather variables. US Department of Agriculture, Agric Res Ser, ARS-8

Schubert S (1994) A weather generator based on the European 'Grosswetterlagen'. Clim Res 4:191-202

Semenov MA, Barrow EM (1997) Use of a stochastic weather generator in the development of climate change scenarios. Clim Change 35:397-414

Semenov MA, Porter JR (1995) Climate variability and the modelling of crop yields. Agric For Meteorol 73:265-283

Semenov M A, Brooks RJ , Barrow EM, Richardson CW (1998) Comparison of WGEN and LARS-WG stochastic weather generators for diverse climates. Clim Res 10:95-107

Sharpley AN, Williams J R (eds) (1990a) EPIC - Erosion/Productivity Impact Calculator: 1. Model documentation. US Department of Agriculture Tech Bull No. 1768

Sharpley AN, Williams J R (eds) (1990b) EPIC - Erosion/Productivity Impact Calculator: 2. User manual. US Department of Agriculture Tech Bull No. 1768

StatSoft, Inc (1997) STATISTICA release 5.1. StatSoft, Inc, Tulsa, OK

Wallis TWR, Griffiths J F (1995) An assessment of the weather generator (WXGEN) used in the erosion/productivity impact calculator. Agric For M eteorol 73:115-133

Wilks DS (1992) Adapting stochastic weather generation algorithms for climate change studies. Clim Change 22:67-84

Wilks DS (1998) Multisite generalization of a daily stochastic precipitation generation model. J Hydrol 210:178-191

Wilks DS (1999) Interannual variability and extreme-value characteristics of several stochastic daily precipitation models. Agric For M eteorol 93:153-169

Submitted: J une 28, 1999; Accepted: December 2, 1999

Proofs received from author(s): February 17, 2000 